\title{
Transcriptome and whole genome sequencing profiles in Leber's Hereditary Optic Neuropathy 14484T $>C$ mutation carrying monozygotic twins reveal that prostanoid receptor is a possible modifier for LHON manifestation
}

Khin Kay Zin Tun

University of Medicine I

Jarichad Toosaranont

Mahidol University Faculty of Medicine Siriraj Hospital

Supannee Kaewsutthi

Mahidol University Faculty of Medicine Siriraj Hospital

Harald Grove

Mahidol University Faculty of Medicine Siriraj Hospital

Sukanya Ruchadaariyachat

Mahidol University Faculty of Medicine Siriraj Hospital

Wichit Suthammarak

Mahidol University Faculty of Medicine Siriraj Hospital

Wanicha Chuenkongkaew

Mahidol University Faculty of Medicine Siriraj Hospital

Patcharee Lertrit

Mahidol University Faculty of Medicine Siriraj Hospital

Bhoom Suktitipat

Mahidol University Faculty of Medicine Siriraj Hospital

Chalermchai Mitrpant ( $\nabla$ chalermchai.mit@mahidol.edu )

Mahidol University Faculty of Medicine Siriraj Hospital https://orcid.org/0000-0001-9311-7977

Primary research

Keywords: LHON carrier, PTGER3, SNPs, mtDNA, reactive oxygen species (ROS), mtDNA haplogroup backgrounds

Posted Date: July 14th, 2021

DOl: https://doi.org/10.21203/rs.3.rs-604500/v1 
License: (c) (i) This work is licensed under a Creative Commons Attribution 4.0 International License. Read Full License 


\section{Abstract \\ Background}

Leber's inherited optic neuropathy (LHON) is well known for incomplete penetrance. A pair of monozygotic twins carrying 14484T > C LHON mutation: one displayed LHON characteristics (affected LHON) and the other twin was an unaffected LHON carrier, were studied to identify possible modifier(s) for LHON manifestation.

\section{Methods}

Primary fibroblasts from affected and unaffected monozygotic twins with 14484T > C LHON mutation were treated with different insults to differentiate cellular phenotype between the two fibroblasts. RNA sequencing of the fibroblasts indicated differentially expressed genes and whole genome sequencing was used to identify candidates for disease modifier.

\section{Results}

Our results suggested that fibroblast from unaffected carrier was able to adapt to galactose and hydrogen peroxide insult, while affected fibroblasts were not. We found reduced expression of total SOD2 with high proportion of inactive SOD2 (acetylated SOD2) in affected LHON fibroblast, while decreased expression of SIRT3 was detected in affected LHON fibroblasts treated with combination of insults. Differential expression indicated enrichment of a pathway relating to negative regulator of cell death pathway in unaffected carrier fibroblast. Expression of receptor for prostaglandin E receptor (PTGER3) was found to be affected by two SNPs. Unaffected LHON fibroblast possessed rs75523942 that indicates a positive effect on PTGER3 expression, while affected LHON fibroblast possessed rs496483 that indicates a negative effect on PTGER3 expression. Discordant SNPs on prostaglandin E receptor 3 (PTGER3) were identified as eQTL.

\section{Conclusions}

This study indicates that prostanoid receptor could be a possible modifier for LHON manifestation of these twins.

\section{Introduction}

Leber's hereditary optic neuropathy (LHON), an inherited mitochondrial disease, typically manifests as painless central visual loss (central scotoma). The visual loss can occur in just one eye or both. Mitochondrial mutations result in death of retinal ganglionic cells (RGCs). LHON is the most common mitochondrial diseases with an estimated prevalence from 1:30,000 to 1:54,000 worldwide. $^{1-3}$ LHON is 
characterized by its incomplete penetrance with gender bias, affecting about $50 \%$ of male mutation carriers and about $10 \%$ of female carriers. ${ }^{4}$ Nearly $95 \%$ of LHON cases are caused by one of the three primary mitochondrial DNA (mtDNA) mutations: $3460 \mathrm{G}>\mathrm{A}$ in MT-ND1, 11778G > A in MT-ND4 or 14484T $>C$ in MT-ND6 subunit of complex $\mathrm{I}^{5-7}$. Recovery of the eyesight in LHON is minimal and clinical severity may depend on type of mutation. Prognosis of patients with $11778 \mathrm{G}>\mathrm{A}$ mutation is the worst while $3460 \mathrm{G}>\mathrm{A}$ and $14484 \mathrm{~T}>\mathrm{C}$ patients display a milder phenotype $\mathrm{e}^{8,9}$.

In earlier studies, a mild to moderate reduction in complex I activity, and complex I dependent ATP synthesis was reported in LHON cybrids carrying either of the above three primary mutations ${ }^{10-13}$. However, LHON cybrids displayed severe ATP depletion and underwent apoptosis in galactose medium indicating bioenergetics insufficiency under OXPHOS stress condition ${ }^{14,15}$. Antioxidant defenses such as glutathione (GSH) content, activities of glutathione reductase (GR), glutathione peroxidase (GPX) or mitochondrial, superoxide dismutase 2 (SOD2) were reduced in LHON cybrids when growing in galactose medium or exposed to hydrogen peroxide $\left(\mathrm{H}_{2} \mathrm{O}_{2}\right)$. These evidences show that cells carrying LHON mutation are vulnerable to oxidative and metabolic stress condition ${ }^{14-16}$.

Therefore, increased reactive oxygen species (ROS) production and insufficient ATP production due to complex I defect have been regarded as major contributing factors to RGCs death in LHON. Like other neurons, RGCs demand the highest energy supply for firing action potential along the axon, especially in unmyelinated part ${ }^{17}$. Competent bioenergetics, along with functional antioxidant system are important to supply adequate ATP as well as prevent oxidative damage to mitochondrial DNAs, lipids and proteins under such conditions.

Compensatory increase in mitochondrial biogenesis of unaffected LHON carriers from European populations was proposed as an underlying factor for incomplete penetrance in $3460 \mathrm{G}>\mathrm{A}$ and $11778 \mathrm{G}>$ $A^{18,19}$. Additionally, mtDNA haplogroup backgrounds, ${ }^{20,21}$ X-linked loci, ${ }^{22,23}$ and potential nuclear modifier gene(s) explored in genome wide studies ${ }^{24-26}$ were reported to influence LHON penetrance though findings varied across populations probably owing to diverse nuclear background in different ethnicities. In addition, environmental factors, such as smoking ${ }^{27}$ and exposure to certain drugs ${ }^{28-30}$ may also trigger LHON expression.

With advances in high-throughput sequencing technologies, comparison of affected LHON patients and unaffected carriers using omics-wide approaches are of great help to identify disease modifying variants ${ }^{31}$. Monozygotic twins supposedly have identical genetic profiles but discrepancy between disease phenotypes may stem from small errors of DNA replication or variation in epigenetic predisposition $^{32,33}$. Consequences of genetic or epigenetic variations could eventually reflect in gene expression, thus comparing transcriptomics of such discordant monozygotic twins will provide invaluable clues to exploration of disease modifying gene(s), associated signaling pathways and regulatory biological processes. We exploited an oxidative and metabolic insult culture in order to demonstrate a difference in cellular phenotype in 14484T >C mutation carrying monozygotic twins, one 
unaffected and one affected by LHON. We then created transcriptomic profiles using next-generation sequencing (NGS) based RNA sequencing (RNA-Seq) on the Illumina platform. Differentially expressed genes were identified and explored to find potentially interplaying signaling pathways and biological processes, which might be beneficial in understanding LHON pathogenesis and application for therapeutic interventions.

\section{Results}

Effect of hydrogen peroxide or galactose medium treatment on wild type and 14484T $>C$ mitochondrial mutation carrying fibroblasts

Primary dermal fibroblasts from normal, unaffected LHON and affected LHON individuals were used to determine cellular phenotype. Treatment of hydrogen peroxide in affected LHON fibroblasts lead to decreased survival compared to normal fibroblasts (Fig. 1A and B). Increased production of reactive oxygen species (ROS) with decreased ATP production were found in $\mathrm{H}_{2} \mathrm{O}_{2}$ treated affected LHON fibroblasts compared to unaffected LHON fibroblasts (Fig. 1C and F). We subsequently treated all three fibroblasts with galactose medium; however, no differences in cell survival and ROS production were found (Fig. 1B and D). However, both unaffected and affected LHON fibroblasts significantly produced less ATP compared to normal fibroblast when cultured in galactose medium (Fig. 1E). Interestingly, combination of hydrogen peroxide and galactose medium treatment on affected LHON fibroblasts led to remarkably reduced survival compared to unaffected LHON and normal fibroblasts, and this finding was consistent with significant increase in ROS production compared to either unaffected LHON or wild type fibroblasts (Fig. 1B and D).

Combination of hydrogen peroxide and galactose medium treatment on affected LHON fibroblasts led to reduced Sirtuin 3 (SIRT3) expression and its functionality on superoxide dismutase 2 (SOD2)

Lower expression of SOD2 in affected LHON fibroblast was noted among culture of three fibroblasts with basal cultured condition (Fig. 2A and B). Treatment with either $\mathrm{H}_{2} \mathrm{O}_{2}$ or galactose medium on affected LHON fibroblasts led to reduced SOD2 expression in affected group (Fig. 2A and B). Combination of hydrogen peroxide and galactose medium treatment on affected LHON fibroblasts further reduced the expression of SOD2 expression compared to either hydrogen peroxide or galactose medium insult; however, a substantial surge of an inactive SOD2 form (lysine acetylation on the 68th amino acid of SOD2) was observed (Fig. 2C). As this Lysine acetylation of SOD2 is known to be regulated by Sirtuin 3 enzyme (SIRT3), we investigated the expression of SIRT3 and found that SIRT3 was marginally reduced in affected LHON fibroblasts treated with combination of the two insults (Fig. 3A and B).

\section{RNA-Seq of monozygotic twins with 14484T > C LHON mutation}


Total RNA extracted from unaffected and affected LHON fibroblasts with four different conditions, i.e. untreated, $\mathrm{H}_{2} \mathrm{O}_{2}$, galactose and a combination of both, were included in the RNAseq study. There were 40,112 transcripts detected from differential expression analysis, as shown in Fig. 4A and B. Five hundred and seventy-seven transcripts were upregulated, while 120 transcripts were downregulated in unaffected LHON fibroblast. Four gene ontologies, relating to negative regulator controlling cell death, were enriched in this gene list at the FDR $<0.05$ (Fig. 4C and D).

\section{Utilisation of whole genome sequencing and whole transcriptome sequencing of $14484 \mathrm{~T}>\mathrm{C}$ mutation carrying fibroblasts to identify a potential discordant SNPs causing disease phenotype}

From DNA samples obtained from buffy coat, we identified 4,913,254 SNVs and small indels presented in unaffected carrier and 4,868,543 SNPs and small indels in affected LHON. In total, there were 263,668 variants present only in unaffected carrier and 219,957 variants present only in affected LHON.

The top twenty differentially expressed gene transcripts, based on adjusted $p$-value, were used to identify discordant SNPs from whole genome sequencing data of the twins. Eighty-three discordant SNPs were identified on 13 different genes in unaffected LHON fibroblast, while 63 discordant SNPs were identified on 13 different genes in affected LHON fibroblast. (Table 1)

The 146 discordant SNPs were investigated against expression quantitative trait loci database, but only four SNPs were present in the database with normalised effect size information (Table 1). Among these four SNPs, three were present in unaffected LHON while one SNP was present in affected LHON fibroblast (Table 2). However, the effect size of only two SNPs, i.e. rs75523942 in unaffected LHON and rs496483 in affected LHON, were in keeping with expression data from RNAseq, and those two were located on prostaglandin E receptor 3 (PTGER3).

\section{Discussion}

Culturing cells with galactose medium forces cells to rely on solely OXPHOS bioenergetics, thereby allowing us to exaggerate cellular phenotypes in diseases with mitochondrial dysfunction ${ }^{34}$. We found that cell viability of $14484 \mathrm{~T}>\mathrm{C}$ mitochondrial mutation carrying LHON affected patient fibroblast was significantly lower than that of unaffected carrier in galactose culture supplemented with hydrogen peroxide. This combination of metabolic insult induced significantly higher level of ROS in affected LHON. This finding was in keeping with the previous report of mitochondrial mutations induced cybrids cells, in which viability of LHON cybrids were significantly reduced as the cells underwent apoptosis in galactose culture after 48 hours of incubation ${ }^{15}$. Increased cellular ROS level along with reduction in cell viability of affected patient fibroblasts in galactose culture under oxidative stress prompted us to determine functionality of the antioxidant system.

We looked into protein expression of the antioxidant enzyme mitochondrial Manganese superoxide dismutase 2 (SOD2) and also lysine (K)-68 acetylated SOD2, an inactive dismutase form. This K68AcSOD2 was also previously reported to sensitise cells to peroxide stress ${ }^{35}$. Our results indicated that 
LHON affected fibroblasts expressed lower level of total SOD2 and that either peroxide, galactose medium or a combination aggravated reduced level of SOD2 expression, while combination of metabolic insults promoted expression of K68-AcSOD2 form, exclusively in affected LHON fibroblast. These findings suggested that aberrant regulation of acetylation switch on SOD2 may occur.

Sirtuin3 (SIRT3) is key regulator of mitochondrial protein acetylation, to curb production of mitochondrial ROS. Several studies showed that SIRT3 reduces ROS levels in order to prevent oxidative stress induced neuronal cell death via increasing activities of antioxidant enzymes activities ${ }^{36-39}$. However, expression of SIRT3 protein was lower in affected, albeit a marginal change. SIRT3 depends on nicotinamide adenine dinucleotide $\left(\mathrm{NAD}^{+}\right)$metabolite to exert its activity. In diseases with mitochondrial dysfunction, conversion of $N A D H$ to $N A D^{+}$is defective, and it can potentially mitigate activity of $N A D^{+}$dependent enzyme. In fact, repletion of $\mathrm{NAD}^{+}$through nicotinamide riboside (NR) supplement can improve mitochondrial biogenesis in mice carrying a dominant pathogenic mutation in the major mitochondrial DNA $^{40}$.

Our RNA sequencing data demonstrated that quinolate phosphoribosyl transferase (qPRT) gene transcript was significantly higher in unaffected LHON with adjusted p-value of 0.0071 , and this may perhaps indicate that unaffected LHON fibroblasts had a better response to replete the pool of NAD ${ }^{+}$ compared to affected LHON fibroblasts. However, the discordant SNPs located in SOD2, STAT3 and QPRT were not associated with eQTL in the GTEx database (https://gtexportal.org) ${ }^{41}$.

Differential expression between unaffected and affected LHON fibroblasts yielded an up and down regulated group of genes enriched on cell death and negative regulator of apoptosis. We subsequently utilised the top twenty of the differentially expressed gene transcript list to search for discordant SNPs as a mean to identify a potential cause of disease phenotype.

Our whole genome sequencing data from twins indicates that discordant SNPs located on CLIC6 and PTGER3 were associated with its expression in GTEx database. CLIC6 is a non-classical ion channel that is structurally homologous to the Glutahione S- transferases Superfamily ${ }^{42}$, and was reported to be highly expressed in human retina ${ }^{43}$. The discordant SNP of CLIC6 present in unaffected twin is negatively associated with its expression. Expression of receptor for prostaglandin E receptor (PTGER3) was found to be affected by two SNPs. Unaffected LHON fibroblast possessed rs75523942 that indicates a positive effect on PTGER3 expression, while affected LHON fibroblast possessed rs496483 that indicates a negative effect on PTGER3 expression. PTGER3 is a prostanoid receptor for a lipidic molecule, so called prostaglandin E2 $\left(\mathrm{PGE}_{2}\right)^{44}$. Knockdown of PTGER3 led to apoptosis, while overexpression of PTGER3 protected myocardium against ischemic reperfusion injury ${ }^{45}$, in which ROS play pivotal role in pathomechanism ${ }^{46,47}$.

\section{Conclusions}


In conclusion, we utilised the preconditioned stress culture to differentiate cellular phenotype of patient with mitochondrial disease from the carrier. Discrepancy of the disease phenotype possibly stem from antioxidant mechanism. Cellular capability of the cells to replete pool of $\mathrm{NAD}^{+}$may also contribute to this cellular phenotype.

\section{Methods}

\section{Sample collection}

Dermal fibroblasts were cultured from a pair of monozygotic twins carrying homoplasmic $14484 \mathrm{~T}>\mathrm{C}$ mutation, and one control individual. One of these twins is an affected LHON patient while the other one does not have a clinical phenotype. The twins were examined by a neuro-ophthalmologist and the control were an independent individual who visited Siriraj Hospital, Thailand for reasons unrelated to eye disease or any other chronic disease conditions. Determination of LHON mutations were done using Polymerase chain reaction-restriction fragment length polymorphism (PCR-RFLP) technique according to Sudoyo et al. ${ }^{48}$. Informed consent was obtained for all the samples recruited and the study was designed to the adherence of Declaration of Helsinki and approved by Siriraj Institutional Review Board (COA: si710/2015), Mahidol University, Bangkok, Thailand.

\section{Primary fibroblast culture}

Fibroblast samples were proliferated in high glucose Dulbecco's Modified Eagle Medium (12800-058, Gibco, Invitrogen) supplemented with $10 \%$ fetal bovine serum, penicillin $(100 \mathrm{U} / \mathrm{ml})$, streptomycin $(100 \mathrm{ug} / \mathrm{ml})$ and amphotericin $B(5 \mathrm{ug} / \mathrm{ml})$, and the fibroblasts were cultured in humidified incubator at $37^{\circ} \mathrm{C}$ with $5 \% \mathrm{CO}_{2}$ supply. Passage number of fibroblasts used for all experiments were between 4-10 in order to avoid aging phenotype. For basal and galactose medium culture condition, glucose free DMEM (D5030, Sigma Aldrich) was supplemented with either $25 \mathrm{mM}$ of glucose or galactose, with supplements used in medium for proliferation. Galactose preconditioning was incubated for 48 hours prior to sample collection. For oxidative stress treatment, fibroblasts were treated with $100 \mathrm{uM}$ of $\mathrm{H}_{2} \mathrm{O}_{2}$ for 90 minutes prior to sample collection.

\section{Determination of cell viability}

Cell viability was determined by MTT (3-(4,5-Dimethylthiazol-2-yl)-2,5- diphenyltetrazolium bromide, a tetrazole) assay (Sigma Aldrich) according to manufacturer's protocol. Briefly, fibroblasts were seeded in triplicate, and cultured in preconditioned high glucose DMEM or $25 \mathrm{mM}$ galactose media for 48 hours. The medium was replaced by fresh medium supplemented with and without $\mathrm{H}_{2} \mathrm{O}_{2}$ treatment for 90 minutes. Number of viable cells was determined by Synergy H1 Hybrid Multiplate Reader (BioTek) at the 570nm absorbance. Three independent experiments were performed. Percentage of cell viability was expressed in a bar graph format.

\section{Determination of cellular ROS content}


Cellular ROS content was determined using hydroethidine (aka. dihydroethidium or DHE) assay (Invitrogen) according to manufacturer's protocol. Briefly, fibroblasts were seeded and treated with different culture condition in the same fashion as the treatment protocol for cell viability assay. Fibroblasts were subsequently stained with $10 \mu \mathrm{M}$ of DHE for $30 \mathrm{~min}$ at $37^{\circ} \mathrm{C}$ in the dark. DHE oxidation was determined by Synergy $\mathrm{H} 1$ Hybrid Multiplate Reader (BioTek) at the excitation/emission of $518 \mathrm{~nm} / 606 \mathrm{~nm}$. Cellular ROS content is expressed as percentage of the DHE oxidation of untreated controls in glucose or galactose medium respectively.

\section{Determination of whole cell ATP content}

Whole cell ATP content was determined by using ATP bioluminescence assay kit (Sigma) according to manufacturer's protocol. Fibroblasts were seeded in precondition of either high glucose DMEM or 25mM galactose media for 48 hours. The medium was replaced by fresh medium supplemented with/without $\mathrm{H}_{2} \mathrm{O}_{2}$ treatment for 90 minutes. Fibroblasts were washed in cold PBS for twice, ATP was extracted in $0.1 \%$ TritonX-100 ATP extraction buffer, as described by Koszegi et al. ${ }^{49}$. Supernatant were kept on ice, stored at $-20^{\circ} \mathrm{C}$ and used for quantification of ATP and protein content. Protein content was determined by Bradford assay (Bio-Rad). Whole cell ATP level was expressed by $\mu \mathrm{mol} / \mathrm{mg}$ protein and percentage of ATP content in galactose was expressed by ratio of whole cell ATP level in galactose medium to that of glucose medium. Percentage of ATP content under oxidative stress was expressed by ratio of whole cell ATP level in $\mathrm{H}_{2} \mathrm{O}_{2}$ exposed cells to that of untreated cells.

\section{Whole cell protein extraction and western blotting}

Fibroblasts cultured in their respective experimental conditions were trypsinised and washed in PBS. Cell pellets were dissociated in RIPA lysis buffer with freshly added protease inhibitors (Sigma) and 1mM of phenylmethylsulfonyl fluoride (PMSF). Extracted protein concentration were measured using Bradford's method and stored at $-80^{\circ} \mathrm{C}$ until use. Whole cell protein extracts were resolved in $10 \%$ SDS-PAGE. The PVDF membrane was blocked in $5 \%$ skimmed milk in $0.1 \%$ Tween-PBS (PBST) buffer for 1 hour to reduce non-specific binding and the blotted membranes were incubated with respective primary antibodies at $4^{\circ} \mathrm{C}$ overnight. The membranes were subsequently incubated with horseradish peroxidase (HRP)conjugated secondary antibody Chemiluminescence HRP solution (Millipore) was applied to visualize target protein bands on the Image Quant LAS 4000 (GE healthcare). Band intensities of proteins were determined using Image $\mathrm{J}$ software (NCBI). Primary and secondary antibodies used were SIRT3 (Cell Signaling Technologies); $\beta$-actin (Sigma); SOD2, Lys-68 acetylated SOD2, goat anti-rabbit and anti-mouse IgG HRP antibodies (Abcam).

\section{RNA extraction and transcriptome profiling}

Total RNA was extracted from fibroblasts grown in basal glucose culture, and culture with hydrogen peroxide, galactose or combination using RNeasy Mini Kit (Qiagen) according to manufacturer's protocol. RNA Sequencing (RNA-Seq) was performed by lllumina using next generation sequencing. FASTQ files were uploaded to a web-based RNA-seq analysis pipeline by Basepair (https://www.basepairtech.com/). 
Short-read data were aligned to the reference genome from UCSC (GRCh37) using STAR aligner ${ }^{50}$ and differential counts obtained from HT-seq count were determined by DESeq2 ${ }^{51}$. List of differentially expressed genes from twins with different conditions were pre-ranked based on $p$-value and log-2 fold change prior to Gene set enrichment analysis (GSEA). GSEA was performed using GSEA software v 4.0.3

\section{DNA extraction and Whole Genome Sequencing (WGS)}

DNA was extracted from whole blood samples and sent for WGS on Illumina's HiSeq-X platform (Macrogen Inc, Korea). The average depth of coverage was 42.2 ( $90.8 \%$ of the genome > 30x). GATKv4-03 best-practice workflow ${ }^{53}$ was used for data processing and variant discovery. Structural variants were checked using Lumpy. We used Variant Effect Predictor (ensembl-VEP version 90.1) ${ }^{54}$ to annotate these discovered variants. The data was analyzed on Firecloud-Terra (https://firecloud.terra.bio/) using GRCh38 for alignment and dbNSFP.v3.5 $\mathrm{a}^{55}$ as the main annotation resource. First, all high impact variants as predicted from VEP's gene model with known clinical significance from ClinVar (https://www.ncbi.nlm.nih.gov/clinvar/) were retained. Then, we kept the moderate impact variants predicted to be deleterious by metaSVM ${ }^{56}$ and Combined Annotation Dependent Depletion (CADD) ${ }^{57}$ score $>15$. Furthermore, we kept only variants with minor allele frequency (MAF) under $1 \%$ from GnomAD ${ }^{58}, 1000$ Genomes $^{59}$, ESP6500, and ExAC databases ${ }^{60}$.

\section{Expression quantitative trait loci (eQTL)}

Transcripts, which were upregulated in unaffected LHON fibroblasts were listed based on adjusted pvalue. Top twenty transcripts were checked against whole genome sequencing data of unaffected and affected LHON samples to identify discordant SNPs. Discordant SNPs in either unaffected or affected LHON fibroblasts were subsequently checked for their expression quantitative trait loci (eQTL) on the GTEx database ${ }^{41}$.

\section{Statistical analysis}

Data obtained from different experiments among study groups were compared between 2 groups by twotailed Student's t-test or Wilcoxon Rank-Sum Test; among study groups by Kruskal Wallis Test or one-way ANOVA test followed by post-hoc Tukey's Test.

\section{Abbreviations}

Leber's hereditary optic neuropathy (LHON), Reactive oxygen species (ROS), expression quantitative trait loci (eQTL), whole genome sequencing (WGS), Adenosine triphosphate (ATP)

\section{Declarations}

\section{Ethical approval and consent to participate}


Informed consent was obtained for all the samples recruited and the study was designed to the adherence of Declaration of Helsinki and approved by Siriraj Institutional Review Board (COA: si710/2015), Mahidol University, Bangkok, Thailand.

\section{Consent for publication}

Not applicable

\section{Availability of data and materials}

The datasets used and/or analysed during the current study are available from the corresponding author on reasonable request.

\section{Competing interests}

Authors have declared no conflict of interest.

\section{Funding}

This project is supported by Mahidol University Grant No. R0015820002 to PL, CM, BS and WS and Mahidol University-Thailand Research Fund (TRF) Grant No. TRG5780206 to BS.

\section{Authors' contributions}

KZT did experiment and data analysis. JT did experiment and data analysis. SK did characterize mitochondrial DNA mutation. HG did whole genome sequencing and transcriptomic data analysis. SR did transcriptomic analysis. WS critically read manuscript. WC diagnosed LHON patients, collected dermal fibroblasts. PL critically read manuscript. BS planned experiment, analysed data and wrote manuscript. CM planned experiment, analysed data and wrote manuscript.

\section{Acknowledgements}

MS. Wipada Promkhuntod was supported by Faculty of Medicine, Siriraj Hospital to facilitate this project.

\section{References}

1. Mascialino B, Leinonen M, Meier T. Meta-analysis of the prevalence of Leber hereditary optic neuropathy mtDNA mutations in Europe. Eur J Ophthalmol. 2012;22:461-5.

2. Rosenberg T, Norby S, Schwartz M, et al. Prevalence and Genetics of Leber Hereditary Optic Neuropathy in the Danish Population. Invest Ophthalmol Vis Sci. 2016;57:1370-5.

3. Yu-Wai-Man P, Griffiths PG, Brown DT, Howell N, Turnbull DM, Chinnery PF. The epidemiology of Leber hereditary optic neuropathy in the North East of England. Am J Hum Genet. 2003;72:333-9.

4. Caporali L, Maresca A, Capristo M, et al. Incomplete penetrance in mitochondrial optic neuropathies. Mitochondrion. 2017;36:130-7. 
5. Huoponen K, Vilkki J, Aula P, Nikoskelainen EK, Savontaus ML. A new mtDNA mutation associated with Leber hereditary optic neuroretinopathy. Am J Hum Genet. 1991;48:1147-53.

6. Johns DR, Neufeld MJ, Park RD. An ND-6 mitochondrial DNA mutation associated with Leber hereditary optic neuropathy. Biochem Biophys Res Commun. 1992;187:1551-7.

7. Wallace DC, Singh G, Lott MT, et al. Mitochondrial DNA mutation associated with Leber's hereditary optic neuropathy. Science. 1988;242:1427-30.

8. Johns DR, Heher KL, Miller NR, Smith KH. Leber's hereditary optic neuropathy. Clinical manifestations of the 14484 mutation. Arch Ophthalmol. 1993;111:495-8.

9. Meyerson C, Van Stavern G, McClelland C. Leber hereditary optic neuropathy: current perspectives. Clin Ophthalmol. 2015;9:1165-76.

10. Brown MD, Allen JC, Van Stavern GP, Newman NJ, Wallace DC. Clinical, genetic, and biochemical characterization of a Leber hereditary optic neuropathy family containing both the 11778 and 14484 primary mutations. Am J Med Genet. 2001;104:331-8.

11. Brown MD, Trounce IA, Jun AS, Allen JC, Wallace DC. Functional analysis of lymphoblast and cybrid mitochondria containing the 3460,11778 , or 14484 Leber's hereditary optic neuropathy mitochondrial DNA mutation. J Biol Chem. 2000;275:39831-6.

12. Brown MD, Zhadanov S, Allen JC, et al. Novel mtDNA mutations and oxidative phosphorylation dysfunction in Russian LHON families. Hum Genet. 2001;109:33-9.

13. Vergani L, Martinuzzi A, Carelli V, et al. MtDNA mutations associated with Leber's hereditary optic neuropathy: studies on cytoplasmic hybrid (cybrid) cells. Biochem Biophys Res Commun. 1995;210:880-8.

14. Baracca A, Solaini G, Sgarbi G, et al. Severe impairment of complex I-driven adenosine triphosphate synthesis in leber hereditary optic neuropathy cybrids. Arch Neurol. 2005;62:730-6.

15. Ghelli A, Zanna C, Porcelli AM, et al. Leber's hereditary optic neuropathy (LHON) pathogenic mutations induce mitochondrial-dependent apoptotic death in transmitochondrial cells incubated with galactose medium. J Biol Chem. 2003;278:4145-50.

16. Schoeler S, Winkler-Stuck K, Szibor R, et al. Glutathione depletion in antioxidant defense of differentiated NT2-LHON cybrids. Neurobiol Dis. 2007;25:536-44.

17. Yu DY, Cringle SJ, Balaratnasingam C, Morgan WH, Yu PK, Su EN. Retinal ganglion cells: Energetics, compartmentation, axonal transport, cytoskeletons and vulnerability. Prog Retin Eye Res. 2013;36:217-46.

18. Bianco A, Valletti A, Longo G, et al. Mitochondrial DNA copy number in affected and unaffected LHON mutation carriers. BMC Res Notes. 2018;11:911.

19. Giordano C, Iommarini L, Giordano L, et al. Efficient mitochondrial biogenesis drives incomplete penetrance in Leber's hereditary optic neuropathy. Brain. 2014;137:335-53.

20. Kaewsutthi S, Phasukkijwatana N, Joyjinda Y, et al. Mitochondrial haplogroup background may influence Southeast Asian G11778A Leber hereditary optic neuropathy. Invest Ophthalmol Vis Sci. 
2011;52:4742-8.

21. Zhang M, Zhou X, Li C, et al. Mitochondrial haplogroup M9a specific variant ND1 T3394C may have a modifying role in the phenotypic expression of the LHON-associated ND4 G11778A mutation. Mol Genet Metab. 2010;101:192-9.

22. Ji Y, Jia X, Li S, Xiao X, Guo X, Zhang Q. Evaluation of the X-linked modifier loci for Leber hereditary optic neuropathy with the G11778A mutation in Chinese. Mol Vis. 2010;16:416-24.

23. Shankar SP, Fingert JH, Carelli V, et al. Evidence for a novel x-linked modifier locus for leber hereditary optic neuropathy. Ophthalmic Genet. 2008;29:17-24.

24. Abu-Amero KK, Jaber M, Hellani A, Bosley TM. Genome-wide expression profile of LHON patients with the 11778 mutation. $\mathrm{Br} \mathrm{J}$ Ophthalmol. 2010;94:256-9.

25. Hudson G, Yu-Wai-Man P, Griffiths PG, et al. Variation in OPA1 does not explain the incomplete penetrance of Leber hereditary optic neuropathy. Mol Vis. 2010;16:2760-4.

26. Phasukkijwatana N, Kunhapan B, Stankovich J, et al. Genome-wide linkage scan and association study of PARL to the expression of LHON families in Thailand. Hum Genet. 2010;128:39-49.

27. Kirkman MA, Yu-Wai-Man P, Korsten A, et al. Gene-environment interactions in Leber hereditary optic neuropathy. Brain. 2009;132:2317-26.

28. Kogachi K, Ter-Zakarian A, Asanad S, Sadun A, Karanjia R. Toxic medications in Leber's hereditary optic neuropathy. Mitochondrion. 2019;46:270-7.

29. Mackey DA, Fingert JH, Luzhansky JZ, et al. Leber's hereditary optic neuropathy triggered by antiretroviral therapy for human immunodeficiency virus. Eye (Lond). 2003;17:312-7.

30. Seo JH, Hwang JM, Park SS. Antituberculosis medication as a possible epigenetic factor of Leber's hereditary optic neuropathy. Clin Exp Ophthalmol. 2010;38:363-6.

31. Jiang $P$, Jin $X$, Peng $Y$, et al. The exome sequencing identified the mutation in YARS2 encoding the mitochondrial tyrosyl-tRNA synthetase as a nuclear modifier for the phenotypic manifestation of Leber's hereditary optic neuropathy-associated mitochondrial DNA mutation. Hum Mol Genet. 2016;25:584-96.

32. Bruder CE, Piotrowski A, Gijsbers AA, et al. Phenotypically concordant and discordant monozygotic twins display different DNA copy-number-variation profiles. Am J Hum Genet. 2008;82:763-71.

33. Huang Y, Ollikainen M, Sipila P, et al. Genetic and Environmental Effects on Gene Expression Signatures of Blood Pressure: A Transcriptome-Wide Twin Study. Hypertension. 2018;71:457-64.

34. Aguer C, Gambarotta D, Mailloux RJ, et al. Galactose enhances oxidative metabolism and reveals mitochondrial dysfunction in human primary muscle cells. PLoS One. 2011;6:e28536.

35. Dikalova AE, Itani HA, Nazarewicz RR, et al. Sirt3 Impairment and SOD2 Hyperacetylation in Vascular Oxidative Stress and Hypertension. Circ Res. 2017;121:564-74.

36. Dai SH, Chen $\mathrm{T}$, Wang $\mathrm{YH}$, et al. Sirt3 protects cortical neurons against oxidative stress via regulating mitochondrial Ca2 + and mitochondrial biogenesis. Int J Mol Sci. 2014;15:14591-609. 
37. Kim SH, Lu HF, Alano CC. Neuronal Sirt3 protects against excitotoxic injury in mouse cortical neuron culture. PLoS One. 2011;6:e14731.

38. Liu L, Peritore C, Ginsberg J, Kayhan M, Donmez G. SIRT3 attenuates MPTP-induced nigrostriatal degeneration via enhancing mitochondrial antioxidant capacity. Neurochem Res. 2015;40:600-8.

39. Shulyakova N, Sidorova-Darmos E, Fong J, Zhang G, Mills LR, Eubanks JH. Over-expression of the Sirt3 sirtuin Protects neuronally differentiated PC12 Cells from degeneration induced by oxidative stress and trophic withdrawal. Brain Res. 2014;1587:40-53.

40. Ryu $D$, Zhang $H$, Ropelle ER, et al. NAD + repletion improves muscle function in muscular dystrophy and counters global PARylation. Sci Transl Med. 2016;8:361ra139.

41. Consortium GT. The Genotype-Tissue Expression (GTEx) project. Nat Genet. 2013;45:580-5.

42. Cromer BA, Morton CJ, Board PG, Parker MW. From glutathione transferase to pore in a CLIC. Eur Biophys J. 2002;31:356-64.

43. Skeie JM, Mahajan VB. Proteomic landscape of the human choroid-retinal pigment epithelial complex. JAMA Ophthalmol. 2014;132:1271-81.

44. Morimoto K, Suno R, Hotta Y, et al. Crystal structure of the endogenous agonist-bound prostanoid receptor EP3. Nat Chem Biol. 2019;15:8-10.

45. Rodriguez-Aguayo C, Bayraktar E, Ivan C, et al. PTGER3 induces ovary tumorigenesis and confers resistance to cisplatin therapy through up-regulation Ras-MAPK/Erk-ETS1-ELK1/CFTR1 axis. EBioMedicine. 2019;40:290-304.

46. Hohlfeld T, Meyer-Kirchrath J, Vogel YC, Schror K. Reduction of infarct size by selective stimulation of prostaglandin EP(3)receptors in the reperfused ischemic pig heart. J Mol Cell Cardiol. 2000;32:28596.

47. Thiemermann C, Zacharowski K. Selective activation of E-type prostanoid(3)-receptors reduces myocardial infarct size. A novel insight into the cardioprotective effects of prostaglandins. Pharmacol Ther. 2000;87:61-7.

48. Sudoyo H, Marzuki S, Mastaglia F, Carroll W. Molecular genetics of Leber's hereditary optic neuropathy: study of a six-generation family from Western Australia. J Neurol Sci. 1992;108:7-17.

49. Koszegi T, Petrik J, Vladimir-Knezevic S, Nagy S. Co-determination of ATP and proteins in Triton X 100 non-ionic detergent-opened monolayer cultured cells. Luminescence. 2007;22:415-9.

50. Dobin A, Davis CA, Schlesinger F, et al. STAR: ultrafast universal RNA-seq aligner. Bioinformatics. 2013;29:15-21.

51. Love MI, Huber W, Anders S. Moderated estimation of fold change and dispersion for RNA-seq data with DESeq2. Genome Biol. 2014;15:550.

52. Subramanian A, Kuehn H, Gould J, Tamayo P, Mesirov JP. GSEA-P: a desktop application for Gene Set Enrichment Analysis. Bioinformatics. 2007;23:3251-3.

53. DePristo MA, Banks E, Poplin R, et al. A framework for variation discovery and genotyping using nextgeneration DNA sequencing data. Nat Genet. 2011;43:491-8. 
54. McLaren W, Gil L, Hunt SE, et al. The Ensembl Variant Effect Predictor. Genome Biol. 2016;17:122.

55. Liu X, Wu C, Li C, Boerwinkle E. dbNSFP v3.0: A One-Stop Database of Functional Predictions and Annotations for Human Nonsynonymous and Splice-Site SNVs. Hum Mutat. 2016;37:235-41.

56. Dong C, Wei P, Jian X, et al. Comparison and integration of deleteriousness prediction methods for nonsynonymous SNVs in whole exome sequencing studies. Hum Mol Genet. 2015;24:2125-37.

57. Rentzsch P, Witten D, Cooper GM, Shendure J, Kircher M. CADD: predicting the deleteriousness of variants throughout the human genome. Nucleic Acids Res. 2019;47:D886-D94.

58. Karczewski KJ, Francioli LC, Tiao G, et al. The mutational constraint spectrum quantified from variation in 141,456 humans. Nature. 2020;581:434-43.

59. Clarke L, Fairley S, Zheng-Bradley X, et al. The international Genome sample resource (IGSR): A worldwide collection of genome variation incorporating the 1000 Genomes Project data. Nucleic Acids Res. 2017;45:D854-D9.

60. Lek M, Karczewski KJ, Minikel EV, et al. Analysis of protein-coding genetic variation in 60,706 humans. Nature. 2016;536:285-91.

\section{Tables}

Due to technical limitations, table 1, 2 is only available as a download in the Supplemental Files section.

\section{Figures}


A
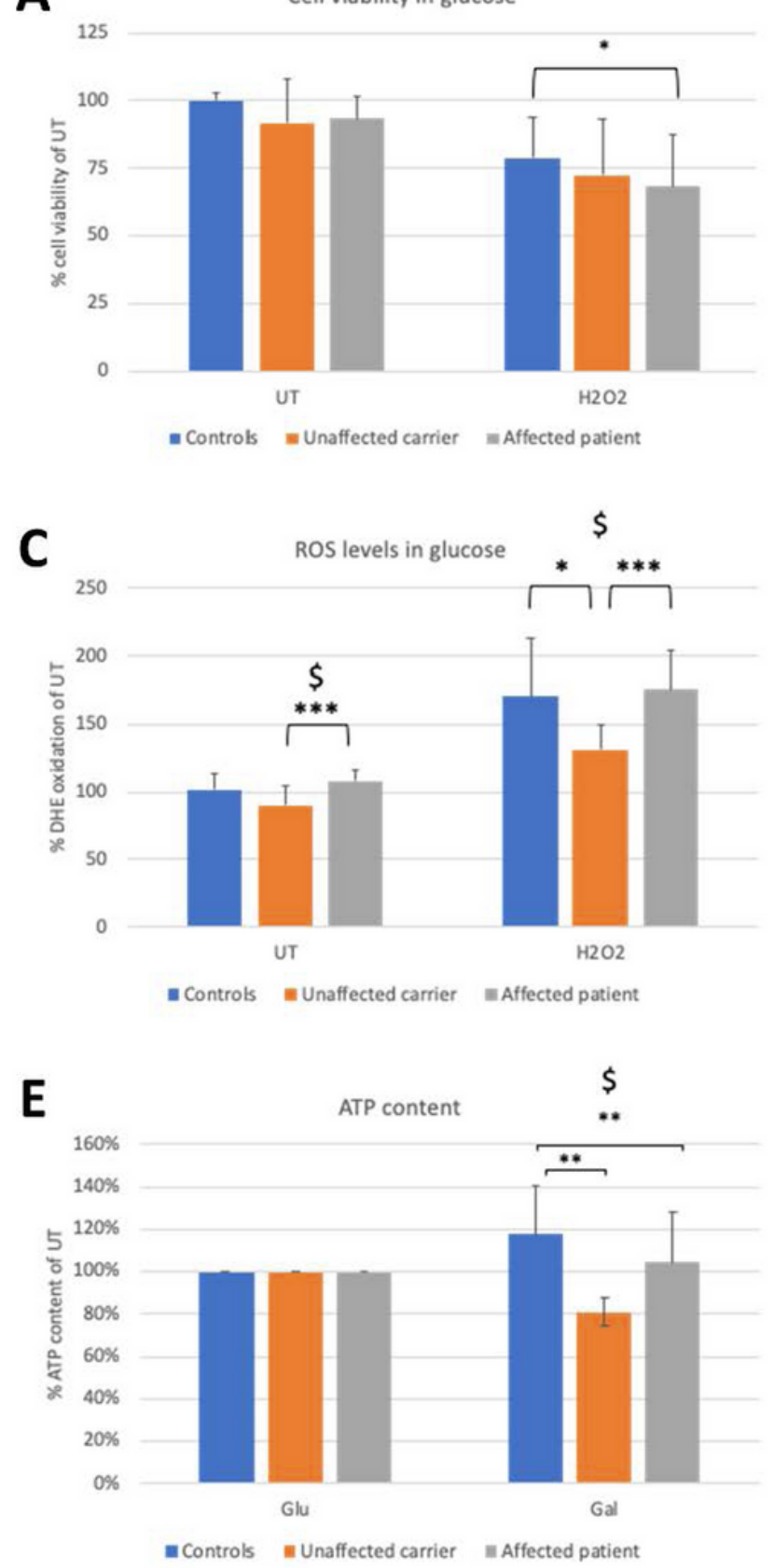

B
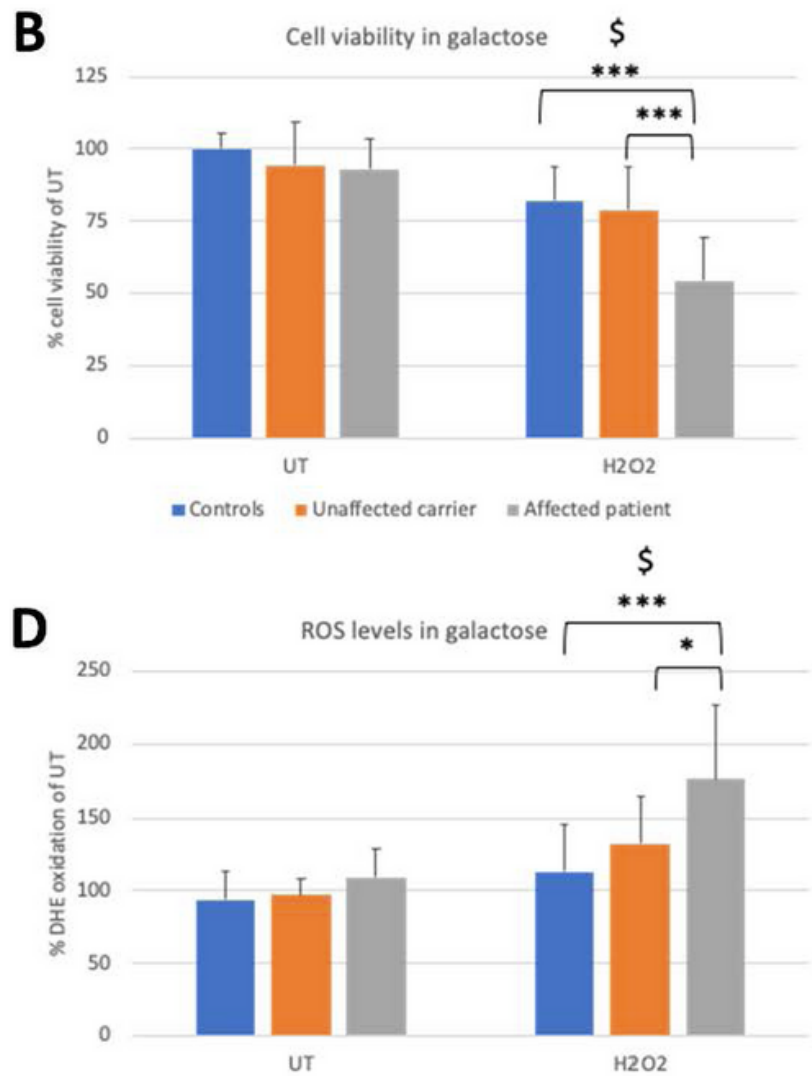

- Controls $\equiv$ Unaffected carrier $\equiv$ Affected patient

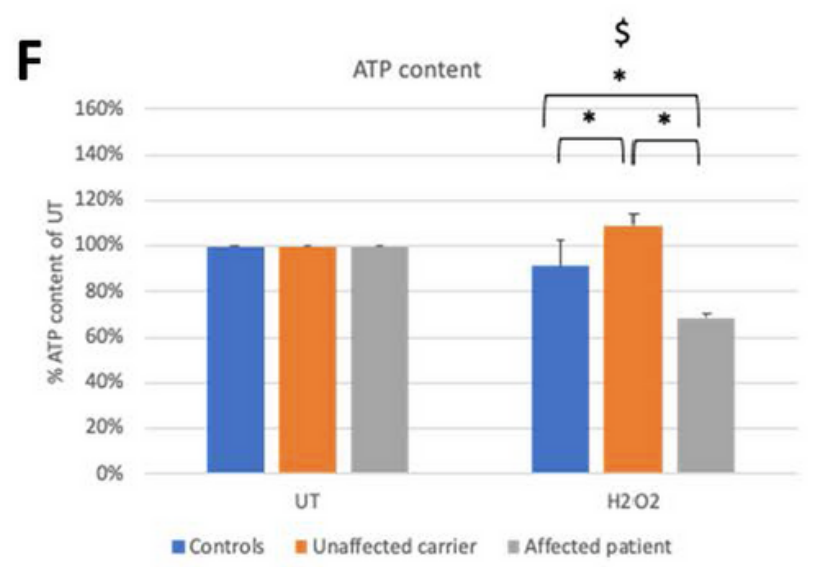

Figure 1

Bar graph demonstrates cell viability (A and B), level of ROS (C and D) or ATP content (E and F) of normal, unaffected LHON and affected LHON fibroblasts, which were treated with galactose, hydrogen peroxide or combination of both metabolic insults. 

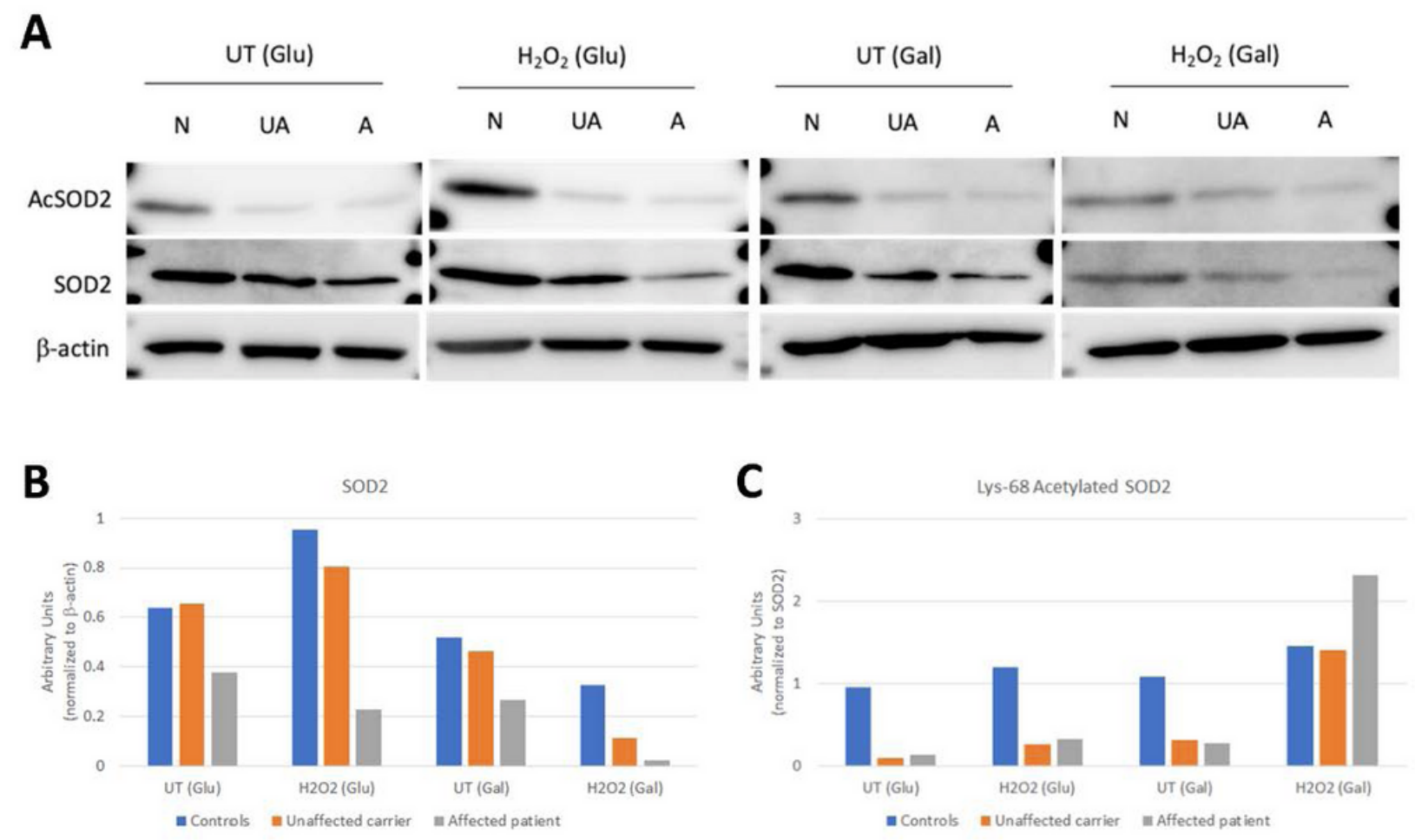

\section{Figure 2}

Western blot analysis of SOD2, acetylated SOD2 at the 68th amino acid of lysine (AcK68-SOD2) and $\beta$ actin (A) on protein extract from normal, unaffected LHON and affected LHON fibroblasts, which were treated with galactose, hydrogen peroxide or combination of both metabolic insults. Densitometric analysis of the protein band of SOD2 that was normalized against $\beta$-actin (B) and AcK68-SOD2 that was normalized against total SOD2 (C). 

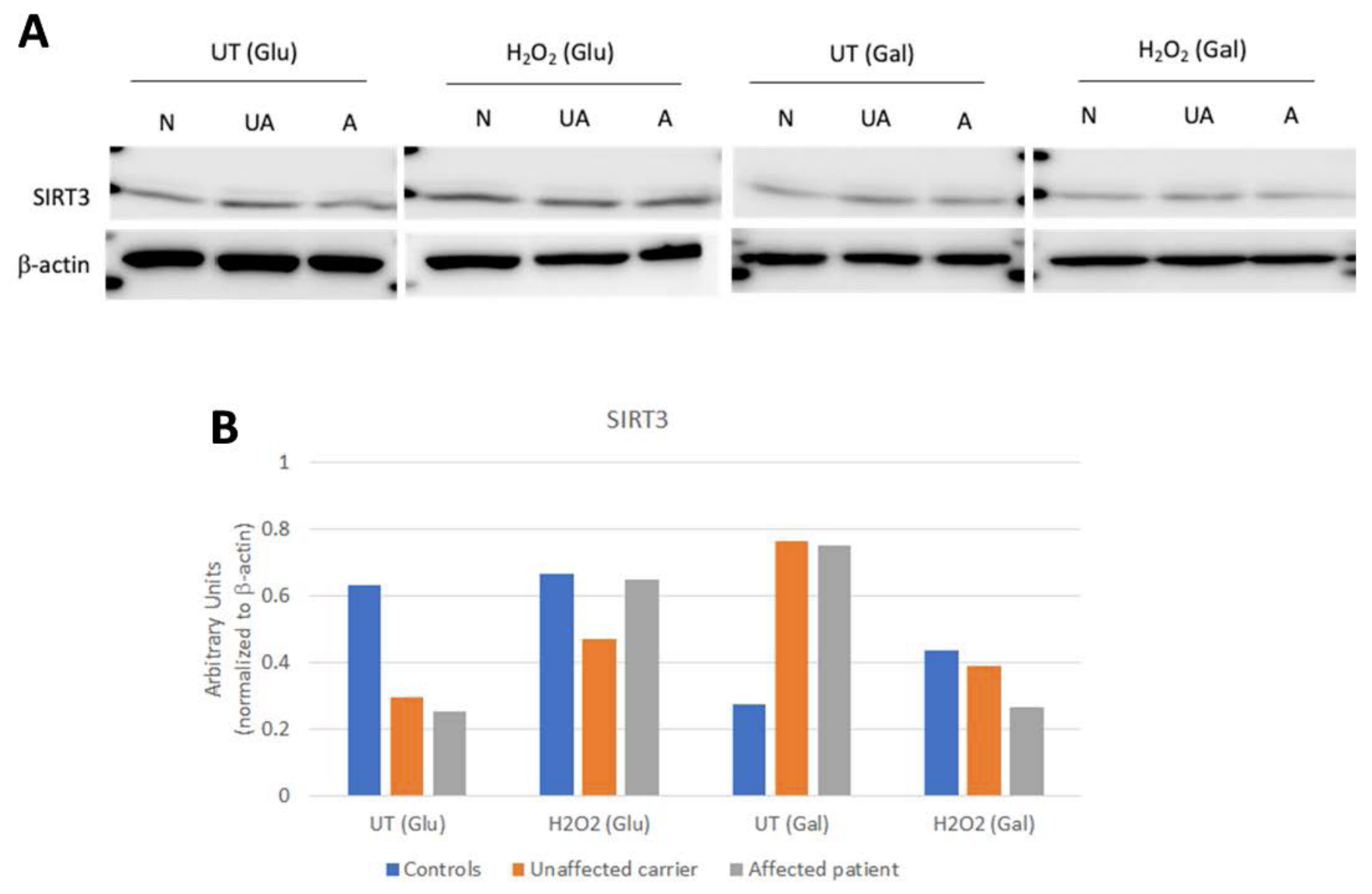

Figure 3

Western blot analysis of SIRT3 and $\beta$-actin (A) on protein extract from normal, unaffected LHON and affected LHON fibroblasts, which were treated with galactose, hydrogen peroxide or combination of both metabolic insults. Densitometric analysis of the protein band of SIRT3 (B) that was normalized against $\beta$ actin. 

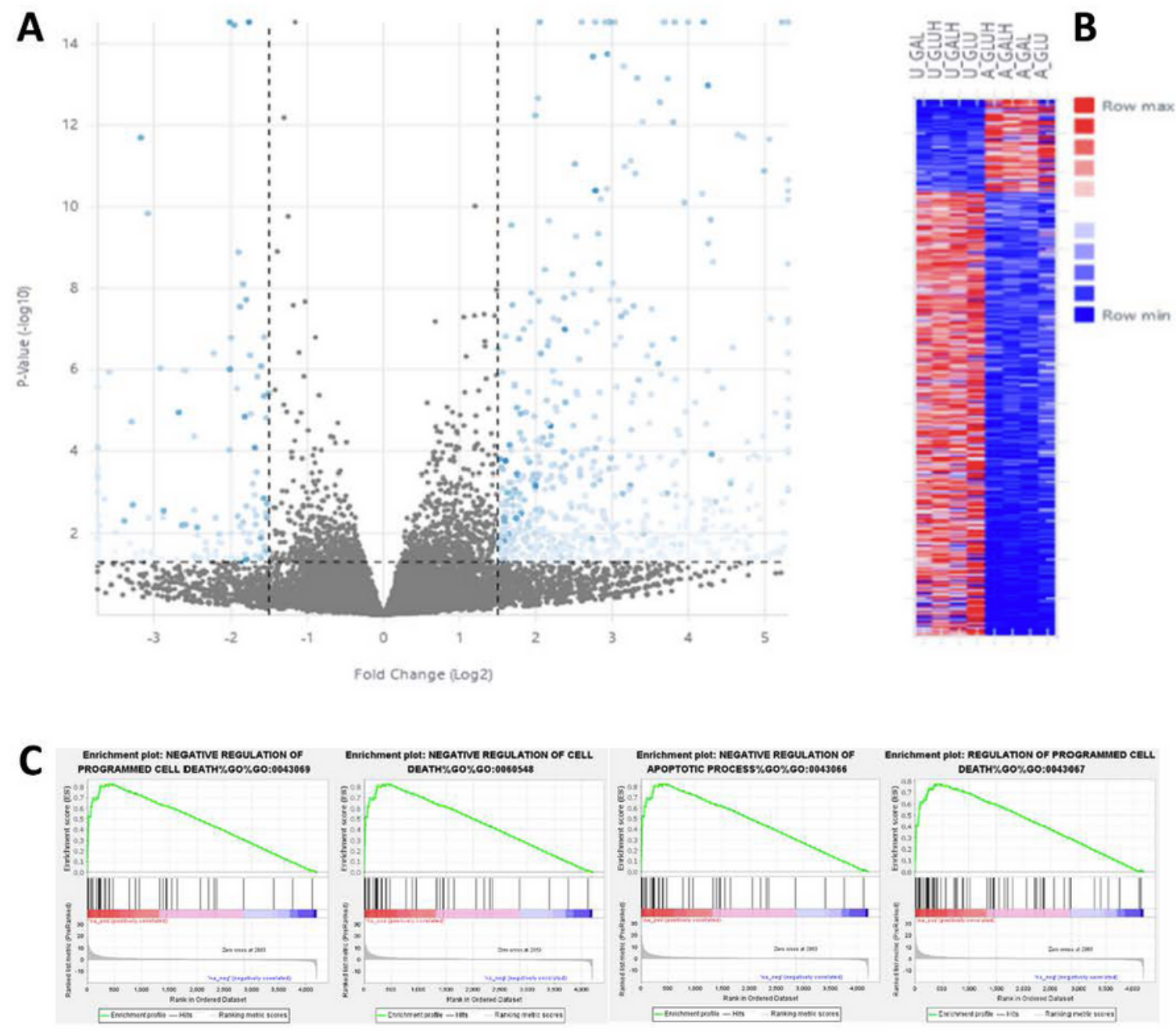

D

\begin{tabular}{|l|c|c|c|c|}
\hline \multicolumn{1}{|c|}{ Gene set enriched followed link to MSigDB } & ES & NES & Nom p-value & FDR q-value \\
\hline NEGATIVE REGULATION OF PROGRAMMED CELL DEATH (GO:0043069) & 0.83 & 1.88 & 0.000 & 0.006 \\
\hline NEGATIVE REGULATION OF CELL DEATH (GO:0060548) & 0.83 & 1.86 & 0.000 & 0.006 \\
\hline NEGATIVE REGULATION OF APOPTOTIC PROCESS (GO:0043066) & 0.83 & 1.86 & 0.000 & 0.004 \\
\hline REGULATION OF PROGRAMMED CELL DEATH (GO:0043067) & & 0.78 & 1.82 & 0.000 \\
\hline
\end{tabular}

\section{Figure 4}

Volcano plot (A) and Heatmap (B) demonstrates a differential expression of gene transcripts between unaffected and affected fibroblasts. Four Gene Ontology pathways were enriched at the false discovery rate $<0.05$. (C and $D)$

\section{Supplementary Files}


This is a list of supplementary files associated with this preprint. Click to download.

- Table1andTable2.pdf 